\title{
Oblique slamming, planing and skimming
}

\author{
S.D. Howison ${ }^{\dagger}$, J.R. Ockendon ${ }^{\dagger}$ and J.M. Oliver* \\ ${ }^{\dagger}$ Mathmatical Institute, 24 - 29 St. Giles', Oxford OX1 3LB; *School of \\ Mathematical Sciences, University of Nottingham, Nottingham NG7 2RD.
}

April 10, 2006

\begin{abstract}
This paper describes how tangential impact velocities can be incorporated into wellknown impact theories in deep and shallow water. Taking the deep and shallow flows in turn, it is shown how to link the normal impact Wagner and Korobkin theories to the tangential impact theories of planing and skimming respectively. An instability is revealed that limits the configurations that can be analysed in the deep water case when the angle of impact is comparable to the deadrise angle. Most of the discussion is confined to two-dimensional flow but a model is also proposed that may describe the spray sheets that can be generated in three-dimensional skimming.
\end{abstract}

Keywords: free-surface flow, inviscid hydrodynamics, ship slamming, water entry, planing.

\section{Introduction}

The phenomenon of violent impact between rigid surfaces and inviscid liquids leads to many interesting models for the evolution of the liquid surface. One that has been intensively studied mathematically is that of slamming, when the normally impacting body is nearly parallel to the initially flat liquid surface (i.e. when the deadrise angle is small). Even with the simplification of two-dimensionality, and neglecting gravity, surface tension and compressibility, the mathematical challenges are formidable. However, progress can be made by using the ideas of codimension-two free boundary problems [1] to formalize Wagner's idea [2] of working with the so-called "contact set" rather than the full wetted region of the impactor $[3,4]$. Crudely speaking, the contact set is the subset of the wetted region over which the pressure is appreciable.

When the liquid is confined by a flat base, a unified sequence of models emerges in terms of the penetration depth [3]. For impact at small deadrise angles the flows can be broadly classified as

- Wagner flows [2] where the penetration depth is much smaller than the layer depth; these flows are characterized by fast thin splash jets emanating from the perimeter of the contact region;

- Korobkin flows [5] where the penetration depth is comparable to the layer depth; these flows are characterized by strong jets, whose thickness is comparable to the liquid depth, but whose roots are still at the perimeter of the contact region. 
Moreover, the sequence of models smoothly joins Wagner flows for small time with Korobkin flows for larger times.

The first aim of this paper is to present a similar hierarchy of models in the case of oblique slamming. When we begin our discussion in $\S 2$, it will soon become apparent that the superposition of a tangential velocity comparable to the normal impact velocity has no effect on the above predictions to lowest order. Indeed, this observation is the resolution of one of Trefethen's paradoxes [6]. Hence, we will focus attention on grazing impact, where the tangential velocity of the impactor is comparable to the speed of the jet roots. However, even within this grazing regime, the range of phenomena is so much broader than for normal impacts that, in this paper, we focus on the modelling challenges rather than their mathematical implications.

In $\S 2$, we begin with the case of semi-infinite liquid regions and we soon encounter grazing impacts where there is the possibility of either cavitation on the "downstream" segment of the contact region, or of the downstream segment effectively exiting the liquid rather than entering it. By using the displacement potential introduced in [7], we will be able to present explicit illustration of effective "downstream exit". Such a configuration would surely lead to the instabilities described in [8] and thereby render any simple free boundary model inappropriate. Hence we regularize the problem by shifting our attention to impactors whose downstream geometry terminates in a sharp corner at which the flow separates smoothly. When the normal impact velocity is negligible, this immediately leads into the regime of planing flows, which have been intensively studied since [9].

There is a famous paradox [9] in the theory of two-dimensional steady planing on liquid of infinite depth, which says that the free surfaces do not have finite asymptotes but instead misalign logarithmically far upstream and downstream; this behaviour is necessary for smooth separation at the trailing edge of the planing body. For small angles of attack, however, the codimension-two idea can be used to think of the flow in terms of a contact region, bounded by the trailing edge and the now small jet root. The index of the associated mixed boundary value problem is one more than in the Wagner theory of slamming; this results in a circulation in the fluid half-space and explains the anomalous far stream free surface behaviour. By using our approach to planing as a regularization of grazing slamming, we will be able to illustrate the idea of [10] that this circulation originates at the "trailing edge" of the planing body at the start of the motion, as in classical unsteady aerofoil theory. We will also be able to see the precise circumstances under which steady planing emerges as the large-time limit.

The above remarks apply to planing bodies that are smooth apart from their trailing edge and at small angles of attack in deep water. As shown in $\S 3$, the same ideas apply when the contact region and water depth are comparable but, in the shallow water Korobkin regime, we will find that new kinds of "spouting" phenomena can occur downstream of the separation point. For negligible normal velocity, this takes us into the skimming regime and we will be able to analyse the circumstances under which Tuck's steady travelling wave solution [11] is approached at large times.

Finally in $\S 4$ we will make some speculations about three-dimensional oblique slamming, planing and skimming. In [4] the three-dimensional generalizations for normal slamming were easy to conjecture and the same is true for grazing with non-negligible normal velocity. However, the strong asymmetry inherent in planing and skimming will now lead to some completely new models. 


\section{Two-dimensional impact on liquid half-spaces}

\subsection{Oblique slamming}

The dimensionless oblique slamming problem is shown in figure 1 in standard notation; $\phi^{*}\left(x^{*}, y^{*}, t\right)$ is the velocity potential and the free surface $y^{*}=h\left(x^{*}, t\right)$ may be multivalued. The body profile is $y^{*}=f\left(\epsilon x^{*}-U t\right)-s(t)$ where $f$ is smooth, $f^{\prime}\left(x^{*}\right) \lessgtr 0$ for $x^{*} \lessgtr 0$, $f(0)=0$ and the small deadrise angle assumption is $\epsilon \ll 1$. The normal impact velocity is $\dot{s}(t)$, where ${ }^{\circ}=\frac{\mathrm{d}}{\mathrm{d} t}$, and the constant tangential velocity is $U / \epsilon$.

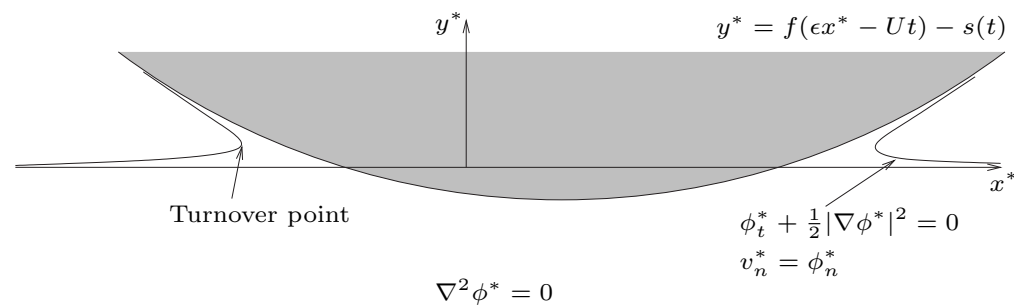

Figure 1: The dimensionless oblique slamming problem. In addition, the kinematic boundary condition on the wetted segment of the body is simply $\phi^{*}{ }_{y^{*}}=-\dot{s}-U f^{\prime}\left(\epsilon x^{*}-U t\right)-$ $\epsilon \phi_{x^{*}}^{*} f^{\prime}\left(\epsilon x^{*}-U t\right)$; the far field conditions are $\phi^{*} \sim \mathrm{O}\left(1 / r^{*}\right)$ as $r^{*} \rightarrow \infty$ (for bounded spatially integrated kinetic energy) and $h \rightarrow 0$ as $\left|x^{*}\right| \rightarrow \infty$; the initial conditions are $\phi^{*}=h=0$ at $t=0$. Subscripts denote partial derivatives, $\phi_{n}^{*}$ is the outward normal derivative of $\phi^{*}$ and $v_{n}^{*}$ is the outward normal velocity of the free surface.

We proceed as in Wagner theory for normal impacts [8] by making the obvious outer scalings $x^{*}=x / \epsilon, y^{*}=y / \epsilon$ and $\phi^{*}=\phi / \epsilon$, relative to which the turnover points clearly have $\mathrm{O}(1)$ speeds. The kinematic boundary condition on the wetted segment of the body $y=\epsilon(f(x)-s(t))$ becomes

$$
\phi_{y}=-\dot{s}-U f^{\prime}(x-U t)-\epsilon \phi_{x} f^{\prime}(x-U t) .
$$

We restrict our attention to order unity time, for which the tangential velocity has an order unity effect through the term $U f^{\prime}(x-U t)$ if and only if $U=\mathrm{O}(1)$, i.e. when the tangential velocity is of the order of the speed of the turnover points predicted by Wagner theory [8]. Hence, for an impactor with $U \ll 1$, the tangential velocity has no leading order effect on Wagner theory in which the leading-order fluid response is as if the body were an expanding flat plate between the turnover points moving with velocity $(0,-1)$. As noted by [12], this explains why in practice an impact splash is largely independent of the tangential velocity. However, in the "grazing" case in which $U=\mathrm{O}(1)$, the standard procedure (see, for example, [4]) results in the leading-order "generalized Wagner problem" in figure 2. The leading-order location of the left $(-)$ and right $(+)$ turnover points are denoted by $x=d_{ \pm}(t)$. The indicated $1 / 2$-power singularities in the potential, and the Wagner conditions that the free surface effectively meets the impactor at $x=d_{ \pm}$, are derived by matching with turnover regions of size $\mathrm{O}\left(\epsilon^{2}\right)$.

Although it is straightforward to write down the solution to the Riemann-Hilbert problem for the complex velocity (see [3]), the resulting integral equations for the free points $x=d_{ \pm}$are complicated. This difficulty can be circumvented by working with the 
displacement potential introduced by [7], namely

$$
\Psi(x, y, t)=-\int_{0}^{t} \phi(x, y, \tau) \mathrm{d} \tau .
$$

Provided that the free points are advancing, we may derive the codimension-two free

$$
\begin{gathered}
\frac{\phi=0, \phi_{y}=h_{t} \quad \phi_{y}=-\dot{s}-U f^{\prime}(x-U t) \quad \phi=0, \phi_{y}=h_{t}}{d_{+}(t)} y=0 \\
d_{-}(t) \\
\nabla^{2} \phi=0
\end{gathered}
$$

Figure 2: Leading-order generalized Wagner problem for grazing entry. In addition, the far field conditions are $\phi \sim \mathrm{O}(1 / r)$ as $r \rightarrow \infty$ and $h \rightarrow 0$ as $|x| \rightarrow \infty$; the initial conditions are $\phi=h=d_{ \pm}=0$ at $t=0$; at the turnover points, $\phi \sim \mathrm{O}\left(r_{ \pm}^{1 / 2}\right)$ as $r_{ \pm}^{2}=\left(x-d_{ \pm}\right)^{2}+z^{2} \rightarrow 0$ and the Wagner conditions are $h\left(d_{ \pm}, t\right)=f\left(d_{ \pm}-U t\right)-s(t)$.

boundary problem for $\Psi$ in figure 3 exactly as in the normal impact case (see, for example, $[7,8])$. The Wagner conditions in figure 2 lead to the boundary condition on the contact set in figure 3.

$$
\begin{gathered}
\Psi=0, \Psi_{y}=-h \quad \frac{\Psi_{y}=s(t)-f(x-U t) \quad \Psi=0, \Psi_{y}=-h}{d_{+}(t)} y=0 \\
d_{-}(t) \quad \nabla^{2} \Psi=0
\end{gathered}
$$

Figure 3: Leading-order generalized Wagner problem for the displacement potential $\Psi$. In addition, the far field conditions are $\Psi \sim \mathrm{O}(1 / r)$ as $r \rightarrow \infty$ and $h \rightarrow 0$ as $|x| \rightarrow \infty$; the initial conditions are $\Psi=h=d_{ \pm}=0$ at $t=0$; at the turnover points $\Psi \sim \mathrm{O}\left(r_{ \pm}^{3 / 2}\right)$ as $r_{ \pm}^{2}=\left(x-d_{ \pm}\right)^{2}+y^{2} \rightarrow 0$, i.e. continuity of the first partial derivatives of $\Psi$ at the free points.

The index of the corresponding Riemann-Hilbert problem for $\Psi_{x}-i \Psi_{y}$ is 3 , so 2 consistency conditions must be satisfied for a unique solution to exist. The conditions at the free points can only be satisfied if

$$
\Psi_{x}-i \Psi_{y}=\sqrt{\left(d_{+}-z\right)\left(z-d_{-}\right)} \int_{d_{-}}^{d_{+}} \frac{s(t)-f(\zeta-U t)}{\sqrt{\left(d_{+}-\zeta\right)\left(\zeta-d_{-}\right)}} \frac{\mathrm{d} \zeta}{\pi(\zeta-z)}
$$

where $z=x+i y$. Hence, expanding as $|z| \rightarrow \infty$, the behaviour at infinity can only be attained if the following two consistency conditions are satisfied:

$$
\int_{d_{-}}^{d_{+}} \frac{[s(t)-f(\zeta-U t)] \zeta^{j}}{\sqrt{\left(d_{+}-\zeta\right)\left(\zeta-d_{-}\right)}} \mathrm{d} \zeta=0 \quad \text { for } \quad j=0,1 .
$$

The physical interpretation of these laws of motion is global conservation of mass $(j=0)$ and $x$-momentum $(j=1)$ and the condition that $\Psi \sim \mathrm{O}(1 / r)$ as $r \rightarrow \infty$ guarantees finite spatially-integrated kinetic energy. 
A straightforward small time analysis shows that for bodies that are blunt at the point of impact, the tangential velocity is negligible at sufficiently small times, the velocities of the turnover points being unbounded as $t \downarrow 0$. Hence, the generalized Wagner theory described in this section applies at sufficiently small times. In contrast, for bodies that are linear on each side of the point of impact, the tangential velocity has a leading-order effect no matter how small the time, the velocities of the turnover points being bounded as $t \downarrow 0$. Hence, it is not clear a priori whether Wagner theory is applicable at sufficiently small times. We now investigate this case further for the wedge $f(x)=|x|$.

\subsubsection{Grazing entry of a wedge}

Assuming $d_{-}<U t<d_{+}$and integrating (4), we find

$$
\begin{gathered}
\pi s=\left(2 U t-d_{-}-d_{+}\right) \sin ^{-1}\left(\frac{2 U t-d_{-}-d_{+}}{d_{+}-d_{-}}\right)+2 \sqrt{U t\left(d_{-}+d_{+}\right)-d_{-} d_{+}-U^{2} t^{2}} \\
\frac{\pi}{2}\left(d_{-}+d_{+}\right) s=\left(\begin{array}{c}
\left.U t\left(d_{-}+d_{+}\right)-\frac{3}{4}\left(d_{-}^{2}+d_{+}^{2}\right)-\frac{1}{2} d_{-} d_{+}\right) \sin ^{-1}\left(\frac{2 U t-d_{-}-d_{+}}{d_{+}-d_{-}}\right) \\
+\left(\frac{3}{4}\left(d_{-}+d_{+}\right)-U t\right) \sqrt{U t\left(d_{-}+d_{+}\right)-d_{-} d_{+}-U^{2} t^{2}}
\end{array}\right.
\end{gathered}
$$

When $s=t$, we seek a similarity solution in the form $d_{ \pm}=a_{ \pm} t$ which leads to (5) and (6) with $s$ and $t$ replaced by 1 and $d_{ \pm}$replaced by $a_{ \pm}$. Figure 4(a) shows a plot of $a_{ \pm}(U)$. The free surface elevation is $h(x, t)=t H(X)$, where $x=X / t$ and

$$
\begin{aligned}
H(X)=-1 & -\frac{2}{\pi} \sqrt{\left(a_{+}-X\right)\left(X-a_{-}\right)} \sin ^{-1}\left(\frac{2 U-a_{+}-a_{-}}{a_{+}-a_{-}}\right) \\
- & \frac{2}{\pi}(X-U) \tan ^{-1}\left(\frac{\left(a_{+}+a_{-}\right)(X+U)-2\left(a_{-} a_{+}+X U\right)}{2 \sqrt{\left(a_{+}-X\right)\left(X-a_{-}\right)\left(a_{+}-U\right)\left(U-a_{-}\right)}}\right),
\end{aligned}
$$

for $X<a_{-}$and $X>a_{+}$. In figure $4(\mathrm{~b})$ we plot $H(X)$ and the wedge profile in the contact set for $U=0,1 / 3,2 / 3,1$. The striking feature is that the trailing turnover point is advancing for $U<U^{*}$ but retreating for $U>U^{*}$, where $U=U^{*} \approx 0.4853$ is the solution to (5) and (6) for which $s=t=1$ and $d_{-}=0$. Hence, the local-in-space and -time linear stability analysis of [8] suggests that the solution is linearly stable if and only if $U<U^{*}$. Since the effective entry velocity $-U f^{\prime}(x-U t)-\dot{s}=-U \operatorname{sgn}(x-U t)-1$ is strictly negative for all $x$ when $U<1$, this instability occurs even though the body is entering on the downstream segment. Moreover, the free surface lies below the wedge when $U<U^{*}$.

This leads us to consider the pressure $p=-\phi_{t}=\Psi_{t t}$. To do so, one must compute the integral of (3) with respect to $z$ except at the free points. We find $p \sim A(U)\left(X-a_{-}\right)^{-1 / 2}$ as $X \downarrow a_{-}$where

$$
A(U)=\frac{1}{\pi}\left[a_{-} \sqrt{\frac{\left(a_{+}-U\right)\left(U-a_{-}\right)}{a_{+}-a_{-}}}-\sqrt{a_{+}-a_{-}} \sin ^{-1}\left(\frac{a_{+}+a_{-}-2 U}{a_{+}-a_{-}}\right)\right] .
$$

We plot $A(U)$ in figure $4(\mathrm{c})$ for $U \in(0.4,0.6)$, which illustrates that $A(U)$ is positive for $U<U^{*}$ but vanishes when $U=U^{*}$. This confirms our view that the asymptotic 

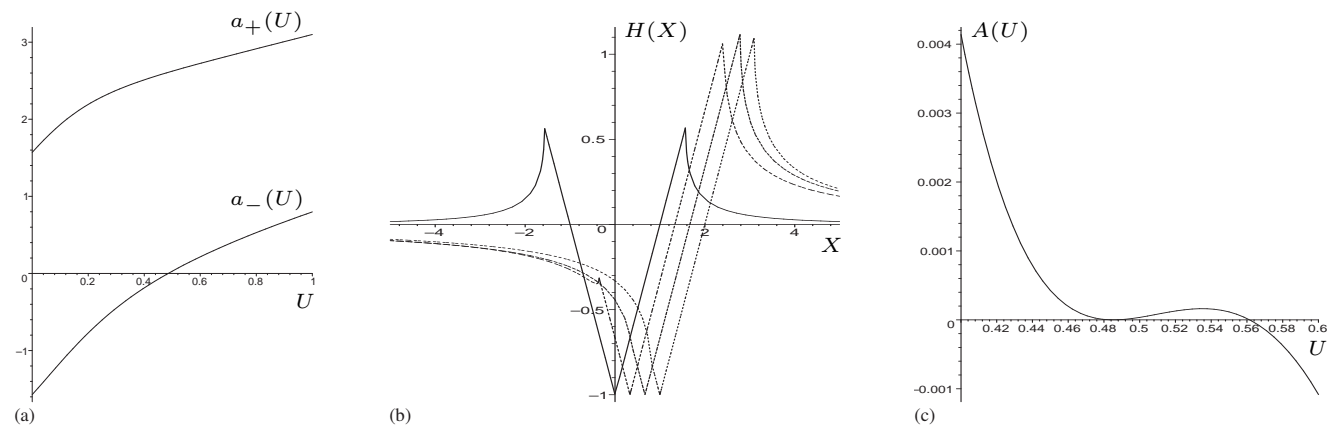

Figure 4: Plot of (a) $a_{ \pm}(U)$, (b) $H(X)$ for $U=0,1 / 3,2 / 3,1$ and (c) $A(U)$ for $U \in$ $(0.4,0.6)$.

decomposition that formally justifies the Wagner theory breaks down as $U \uparrow U^{*}$. However, we have no explanation for the lack of monotonicity in figure 4(c).

Finally, we note that there are no solutions to (5) and (6) of the form $s \sim s_{0}, d_{ \pm} \sim$ $U t \pm b_{ \pm}$as $t \rightarrow \infty$, where $s_{0}, b_{ \pm}>0$ are constants. Hence the solution cannot tend to a steady state for any $s(t)$.

\section{$2.2 \quad$ Planing}

To avoid the nebulous instability problems in the above theory for small incidence angles, we remove the entire downstream segment of the body in $x<U t$ and demand that the free surface separates smoothly from the body at the trailing edge $(x, y)=(U t,-s(t))$. We move to a frame of reference fixed with respect to the horizontal motion of the body by setting $x=U t+\xi$ and $d_{+}=U t+d$, and we subtract the uniform far field flow by setting $\phi=-U x+\hat{\phi}$. The analogue of the codimension-two grazing model in figure 2 is shown in figure 5, in which we have dropped the hat on the potential. As usual the Wagner condition is obtained by matching with the outer region at the turnover point and this matching implies the $1 / 2$-power singularity in $\phi$ in figure 5 . However, the smooth separation condition at the trailing edge has to comply with the well-known inverse square root curvature singularity in the outer free surface at the separation point [13] and this leads to the $3 / 2$-power singularity in $\phi$ in figure 5 .

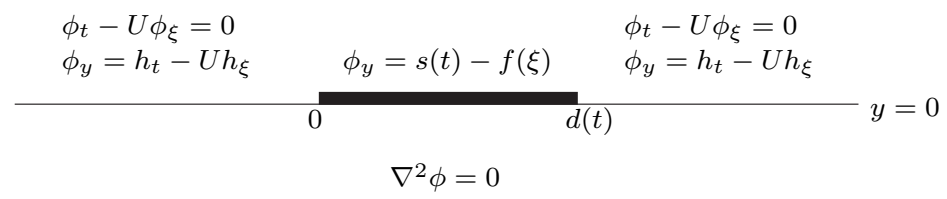

Figure 5: Leading-order problem for infinite-depth unsteady planing. At the trailing edge $\phi \sim \mathrm{O}\left(r^{3 / 2}\right)$ as $r^{2}=\xi^{2}+y^{2} \rightarrow 0$ and the smooth separation condition is $h(0, t)=-s(t)$; at the free point $\phi \sim \mathrm{O}\left(r_{+}^{1 / 2}\right)$ as $r_{+}^{2}=(\xi-d)^{2}+y^{2} \rightarrow 0$ and the Wagner condition is $h(d, t)=f(d)-t$; the far-field conditions are discussed in the text; the initial conditions are $\phi=h=d=0$ at $t=0$.

Seeking a steady state solution in which $s=s_{0}=$ const. is given and $\phi=\phi(\xi, y)$, $h=h(\xi)$ and $d=d_{0}=$ const. are to be found, we find that $\phi_{\xi}(\xi, 0)=0$ on $\xi<0, \xi>d_{0}$. 
Hence, the index of the associated mixed boundary value problem for $\phi_{\xi}-i \phi_{y}$ is 2 and it is impossible to satisfy the condition that the potential is zero in the far field. Writing $z=\xi+i y$, the solution is the classical aerofoil flow

$$
\phi_{\xi}-i \phi_{y}=\frac{U}{\pi} \sqrt{\frac{z}{z-d_{0}}} \int_{0}^{d_{0}} \sqrt{\frac{\zeta-d_{0}}{\zeta}} \frac{f^{\prime}(\zeta)}{\zeta-z} \mathrm{~d} \zeta
$$

with a line vortex of strength $\Gamma=2 U \int_{0}^{d_{0}} \sqrt{\frac{\zeta-d_{0}}{\zeta}} f^{\prime}(\zeta) \mathrm{d} \zeta$ at infinity, i.e.

$$
\phi \sim-\Re\left(\frac{i \Gamma}{2 \pi} \log z\right) \quad \text { as } \quad|z| \rightarrow \infty .
$$

The circulation in the far field leads to Green's paradox [9] that the free surface tends to minus infinity logarithmically at infinity, viz. $h(\xi) \sim-\frac{\Gamma}{2 \pi U} \log |\xi|$ as $|\xi| \rightarrow \infty$. Hence, the linearization of the free surface boundary conditions onto the $\xi$-axis breaks down sufficiently far from the origin. Moreover, the absence of a reference level makes it impossible to invoke the Wagner condition to find $d_{0}$. Hence, there is a one-parameter family of solutions and, as noted by [14], this is an intrinsic feature of steady two-dimensional infinite-depth planing without gravity. Several authors, including [15, 16], have shown that introducing gravity in the far field removes Green's paradox and enables $\Gamma$, and therefore the solution, to be uniquely determined by matching.

In the unsteady case, we must demand zero far field conditions, so the index of the associated mixed boundary value problem for $\phi_{\xi}-i \phi_{y}$ is forced to be 1 for a unique solution to exist. Integrating the pressure boundary condition $\phi_{t}-U \phi_{\xi}=0$ on $y=0$ for $\xi<0$ and $\xi>d$, and applying the initial conditions and continuity of $\phi$ at the trailing edge, we find

$$
\phi(x, 0, t)=\left\{\begin{array}{cl}
0, & x<-U t, x>d \\
\phi(0,0, x / U+t), & -U t<x<0
\end{array}\right.
$$

Since $\phi$ is harmonic in $y<0$ and has specified singularities at the trailing edge, the turnover point and infinity with indices $3 / 2,1 / 2$ and 0 , respectively, there must be an additional singularity or distribution thereof which contributes -1 to the index. One possibility is to introduce a line vortex at $(x, y)=(-U t, 0)$. However, [10] have proposed a trailing vortex sheet on $y=0$ for $-U t<\xi<0$. Either scenario prompts the conjecture that, if $s(t) \rightarrow s_{0}$ as $t \rightarrow \infty$, then the solution to the unsteady planing problem converges to the above steady state solution with a circulation $\Gamma$ at infinity as above, and $d(t) \rightarrow d_{0}$ as $t \rightarrow \infty$. Finally, we remark that, regardless of the chosen approach, the unsteady planing problem in figure 5 is more difficult to solve than the grazing problem in figure 2 because (8) implies that a non-trivial variational formulation as in figure 3 is no longer possible.

\section{Two-dimensional impact on liquid of finite depth}

We now consider the effect of introducing a base at $y^{*}=-H^{*}$ on the grazing and planing models of $\S 2$. The liquid depth $H^{*}$ is an additional length scale and it is natural to characterise flows by comparing $H^{*}$ with the sizes of the penetration depth $s$ and the length of the contact set $L^{*}$. As discussed in [4], we can now distinguish four cases, in 
all of which the deadrise or incidence angle $\epsilon$ is small. In case $(1), s \ll L^{*} \ll H^{*}$, and the effect of the base is not felt to leading order, so the infinite-depth theory applies; this regime also describes the initial stage of the entry of any non-flat impactor. In case (2), we still have $s \ll H^{*}$, but now $L^{*} / H^{*}=O(1)$, so the effect of the base is felt at leading order. Case (3) is the large aspect ratio version of case (2), and occurs if $s \ll H^{*}$ but $L^{*} \gg H^{*}$, and lastly, in case (4), the penetration depth is $O\left(H^{*}\right)$ and the contact length is much greater than $H^{*}$; this is the Korobkin flow regime.

As in the infinite depth case discussed in $\S 2.1$, there is a sharp contrast in the small time asymptotics depending on whether the body is blunt or not at the point of impact. First, we recall that for normal impact of a symmetric body that is smooth except possibly at the point of impact, cases (1)-(4) occur sequentially, and the evolution in each may in general be matched to its predecessor, thereby providing its initial conditions, and/or to its successor, thereby providing the initial conditions of its successor. If the body is blunt, the same is true for grazing impacts in each of cases (1)-(4) because the tangential velocity has no leading-order effect at sufficiently small times. However, for grazing bodies such as the wedge as in $\S 2.1 .1$, the tangential velocity has a leading-order effect no matter how small the time and it is therefore necessary to ensure that the solution in any earlier stages are physically acceptable. Since the theory in case (1) was discussed in $\S 2.1$, we will now consider cases (2)-(4) in order.

\subsection{Oblique slamming}

- Case (2)

In case (2), we suppose $H^{*}=H / \epsilon$ where $H=\mathrm{O}(1)$, so that the layer depth and contact set length are comparable. Hence, the Wagner theory applies and the "case (2) grazing model" is exactly as in the infinite depth case in figure 3 except with a base at $y=-H$. Employing the displacement potential (1) allows us to write down the solution to the associated mixed boundary value problem for $\Psi_{x}-i \Psi_{y}$ by conformally mapping the strip $-H<y<0$ onto the lower half-plane. The index is 3 , as in the infinite depth case, so the unique solution is

$$
\begin{aligned}
\Psi_{x}-i \Psi_{y}=\sqrt{\left(\mathrm{e}^{\pi d_{+} / H}-\mathrm{e}^{\pi z / H}\right)\left(\mathrm{e}^{\pi z / H}-\mathrm{e}^{\pi d_{-} / H}\right) \mathrm{e}^{-\pi z / H}} \times \\
\int_{d_{-}}^{d_{+}} \frac{s(t)-f(\zeta-U t)}{\sqrt{\left(\mathrm{e}^{\pi d_{+} / H}-\mathrm{e}^{\pi \zeta / H}\right)\left(\mathrm{e}^{\pi \zeta / H}-\mathrm{e}^{\pi d_{-} / H}\right) \mathrm{e}^{-\pi \zeta / H}}} \frac{\mathrm{d} \zeta}{\pi(\zeta-z)},
\end{aligned}
$$

provided the following two consistency conditions are satisfied:

$$
\int_{d_{-}}^{d_{+}} \frac{(s(t)-f(\zeta-U t)) \mathrm{e}^{\pi(j+1) \zeta / 2 H}}{\sqrt{\left(\mathrm{e}^{\pi d_{+} / H}-\mathrm{e}^{\pi \zeta / H}\right)\left(\mathrm{e}^{\pi \zeta / H}-\mathrm{e}^{\pi d_{-} / H}\right)}} \mathrm{d} \zeta=0 \quad \text { for } \quad j=0,1 .
$$

The initial conditions are

$$
\phi=d_{ \pm}=0 \quad \text { at } \quad t=0
$$

at least for blunt bodies. The laws of motion (10) have exactly the same physical interpretation as in the infinite depth case in $\S 2.1$. We note that, as $H \rightarrow \infty$ in (9) and (10), we obtain the infinite depth versions (3) and (4), respectively, with an $\mathrm{O}(1 / H)$ error. 
- Case (3)

Case (3) is the small $H$ limit of case (2). Expanding (10) as $H \rightarrow 0$, we find

$$
\int_{d_{-}}^{d_{+}}(s(t)-f(\zeta-U t)) \zeta^{j} \mathrm{~d} \zeta=\mathrm{O}(H) \quad \text { for } \quad j=0,1,
$$

which can be interpreted as conservation of mass $(j=0)$ and horizontal momentum $(j=1)$. These global conditions are consistent with the details of the flow field, which may be found by expanding the displacement potential given by (9) as $H \rightarrow 0$. However, as shown in detail in [3], it is much easier to seek the asymptotic solution of the codimensiontwo free boundary problem in figure 3 with a base at $y=-H$ in the small- $H$ limit; a similar procedure is found in [1] for other codimension-two free boundary problems. This reveals that the flow in the interior region beneath the impactor and between the turnover points may be thought of as an 'inviscid squeeze film flow'. This flow must be matched to the exponentially small flow in the exterior regions outside the turnover points through small transition regions of size $\mathrm{O}(H)$ in the neighbourhood of the turnover points; in these regions the flow is a travelling wave to lowest order. The resulting "case (3) grazing model" for the leading-order squeeze film potential $\phi(x, t)$ (scaled with $1 / H^{2} \epsilon$ ) is the following "codimension-one" free boundary problem:

$$
\phi_{x x}=\dot{s}(t)+U f^{\prime}(x-U t) \quad \text { in } \quad d_{-}<x<d_{+},
$$

with "Rankine-Hugoniot" boundary conditions

$$
\phi=0, \phi_{x}=\dot{d}_{ \pm}(f(x-U t)-s(t)) \quad \text { on } \quad x=d_{ \pm},
$$

and the initial conditions (11), at least for blunt bodies. The consistency condition for (13) and (14) is

$d_{+}-d_{-}+U\left(f\left(d_{+}-U t\right)-f\left(d_{-}-U t\right)\right)=\dot{d}_{+}\left(f\left(d_{+}-U t\right)-s(t)\right)-\dot{d}_{-}\left(f\left(d_{-}-U t\right)-s(t)\right)$,

which, together with (11), imply the two laws of motion (12). We remark that the displacement potential must now be defined as

$$
\Psi=-\int_{0}^{t} \phi(x, \tau) \mathrm{d} \tau .
$$

Then, by integrating (13) and (14) or by carefully taking the small $H$ limit of the displacement potential problem in figure 3 with a base at $y=-H$, we obtain the novel free boundary problem

$$
\Psi_{x x}=s(t)-f(x-U t) \text { for } d_{-}<x<d_{+} \quad \text { with } \Psi=0, \Psi_{x}=0 \text { on } x=d_{ \pm},
$$

which leads, after two integrations, to algebraic equations for $d_{ \pm}(t)$.

\section{- Case (4)}

In case (4), the penetration depth is comparable to the layer depth and we suppose $H^{*}=1$, without loss of generality. Now the squeeze film effect is so strong that the Wagner theory does not apply. In particular the fast moving jets are now comparable 


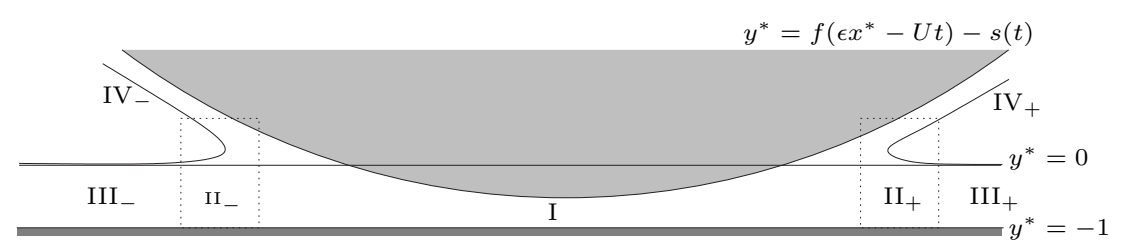

Figure 6: Asymptotic decomposition for the shallow water grazing of a body at small deadrise angle relative to the original (starred) dimensionless variables in figure 1 . The labels that denote the regions are as follows: I :Interior region; $\mathrm{II}_{ \pm}$: Exterior regions; $\mathrm{III}_{ \pm}$: Jet root or turnover regions; $\mathrm{IV}_{ \pm}$: Jet regions.

in thickness to the layer depth. Korobkin's shallow water slamming theory [5] can easily be generalized to grazing impact and the flow field decomposes into the seven regions in figure 6 .

As described in detail in [4], the flow is exponentially small in the exterior regions and matching with the inviscid squeeze film flow in the interior region through the jet roots leads to the following "case (4) grazing model" for the leading-order squeeze film. Scaling $x^{*}=x / \epsilon$ and $\phi^{*}=\phi / \epsilon^{2}$, we find that the velocity potential $\phi(x, t)$ satisfies

$$
F_{t}+\left(F \phi_{x}\right)_{x}=0 \quad \text { in } \quad d_{-}<x<d_{+}
$$

with

$$
\phi=0, \phi_{x}=2 \dot{d}_{ \pm}\left(1-F^{-1 / 2}\right) \quad \text { on } \quad x=d_{ \pm},
$$

where the base-to-body depth is $F(x, t)=1+f(x-U t)-s(t)$. As in case (3), the initial conditions are (11), at least for blunt bodies, and the consistency condition implies conservation of mass and $x$-momentum, though now both these quantities are lost to the jets to lowest order. Also as in case (3), we can integrate (15) twice, but this now leads to coupled ordinary differential equations for $d_{ \pm}(t)$, because we are not able to work with the displacement potential.

\section{$3.2 \quad$ Planing, skimming and spouting}

At small enough angles of incidence, the "case (2) planing model" is exactly as in the infinite depth case in figure 5 except with a base at $y=-H$. This leads to the open problem concerning downstream separation, as discussed in $\S 2.2$ for the infinite depth case. However, it is straightforward to write down the steady state solution by solving the Riemann-Hilbert problem for $\phi_{x}-i \phi_{y}$ and, to lowest order, it is in agreement with the small incidence angle asymptotics of the exact solution of Green [9]. The same is true for the small- $H$ asymptotics in case (3). However, in case (4), when the penetration depth is comparable to the layer depth, the flow behind the planing body is so violent as to lead to a 'spout' as depicted in figure 7. In $§ 3.2 .1$, we describe this skimming regime in detail and proceed in $\S 3.2 .2$ to analyse the special case of the zero-deadrise angle impact of a finite flat plate on shallow water.

\subsubsection{Skimming}

In case (4), we propose that the flow is decomposed into the nine regions in figure 7 . The 


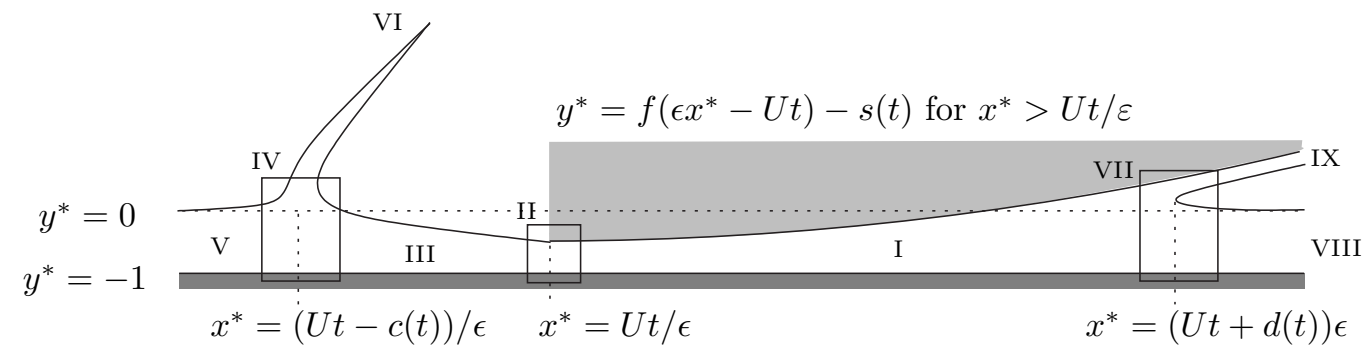

Figure 7: Asymptotic structure of the unsteady skimming problem relative to the original (starred) dimensionless variables in figure 1. To lowest order the flow in each region is as follows. I: Inviscid squeeze film flow; II: Uniform flow; III: Zero-gravity shallow-water flow; IV: Kelvin-Helmholtz flow; V: Exponentially small flow; VI: Thin spout jet governed by zero-gravity jet equations; VII: Kelvin-Helmholtz flow; VIII: Exponentially small flow; IX: Thin spray jet governed by zero-gravity shallow-water equations.

flow ahead of the trailing edge is governed by Korobkin's shallow water impact theory [5] as used in $\S 3.1$. Moving to a frame of reference fixed with respect to the horizontal motion of the body by setting $x=U t+\xi, \phi=U x+\tilde{\phi}, d_{+}=U t+d,(15)$ implies that the flow in region $\mathrm{I}$ is governed by

$$
\tilde{F}_{t}+\left(\tilde{F} \tilde{\phi}_{\xi}\right)_{\xi}=0 \text { for } 0<\xi<d
$$

while the free boundary conditions (16) become

$$
\tilde{\phi}=-U \xi, \tilde{\phi}_{\xi}=U\left(1-2 \tilde{F}^{-1 / 2}\right)+2 \dot{d}\left(1-\tilde{F}^{-1 / 2}\right) \quad \text { at } \quad \xi=d,
$$

where the base-to-body height is $\tilde{F}(\xi, t)=1+f(\xi)-s(t)$. The corresponding pressure is $\frac{1}{2}\left(U^{2}-\tilde{\phi}_{\xi}^{2}\right)-\tilde{\phi}_{t}$, which must vanish at $\xi=0$, i.e. we require that

$$
\tilde{\phi}_{t}+\frac{1}{2} \tilde{\phi}_{\xi}^{2}=\frac{1}{2} U^{2} \quad \text { at } \quad \xi=0
$$

in order to match with the region II, where the smooth separation condition implies that the flow is uniform to lowest order. Except for the initial conditions, this closes the codimension-one free boundary problem for the flow in region I.

In region III behind the impactor, the ejected stream satisfies the 'zero-gravity shallow water' equations. Writing $\phi=U x+\hat{\phi}, h=\hat{h}$, these become

$$
\hat{\phi}_{t}+\frac{1}{2} \hat{\phi}_{\xi}^{2}=\frac{1}{2} U^{2} \text { and } \hat{h}_{t}+\left(\hat{h} \hat{\phi}_{\xi}\right)_{\xi}=0 \quad \text { for } \quad-c(t)<\xi<0
$$

where the spout root $\xi=-c(t)$ is defined below and the boundary data is

$$
\hat{\phi}(0, t)=\tilde{\phi}(0, t), \hat{h}(0, t)=\tilde{F}(0, t)=1-s(t) .
$$

The horizontal stream in region III collides with the initially quiescent layer in region $\mathrm{V}$, which we expect to move exponentially slowly with respect to the fixed frame, as in region VIII. As described in detail in [17], the interaction that occurs is a classical jet impact 
problem in region IV near $\xi=-c(t)$, and this creates a spout region VI. The condition of zero pressure on either side of the spout gives the Rankine-Hugoniot condition

$$
\lim _{\xi \downarrow-c(t)}\left(U+\hat{\phi}_{\xi}(\xi, t)-\dot{c}\right)=\dot{c}, \text { i.e. } \dot{c}=\frac{1}{2}\left(U+\hat{\phi}_{\xi}(c(t), t)\right) .
$$

The spout itself, which is a jet whose thickness may be calculated as in [17], follows a ballistic path from $(-c(t), 0)$, the speed of ejection being equal to $\frac{1}{2} \dot{c}$ and the initial thickness and direction being determined by conservation of mass and horizontal momentum in the Kelvin-Helmholtz flow in the spout region IV.

The theory returns us to the problem mentioned at the beginning of $\S 3$ concerning the initiation of the flow. For blunt impactors with $f^{\prime}(0+)=0$, the velocity of the turnover point is unbounded as $t \rightarrow 0$ and therefore the tangential velocity is negligible at sufficiently small times. Hence, for a complete temporal decomposition one must solve the case (2) planing problem with zero tangential velocity and match with the above solution. If, as for a flat plate impacting at a small, non-zero incidence angle, $f^{\prime}(0+)>0$, the velocity of the turnover point is finite as $t \rightarrow 0$, so one must solve the case (2) planing problem with finite tangential velocity. Hovever, if one assumes, as in Wagner theory, that the $x^{*}$-coordinate of the turnover point scales with the $x^{*}$-coordinate of the point of intersection of the planing body with the undisturbed free surface, then one would expect $c(0)=d(0)=0$.

Finally, we note that the steady state version of $(17-19)$ with $s=s_{0}=$ const. and $d=d_{0}=$ const. is exactly the model that [11] used to analyse the flow past a twodimensional skimming plate at small incidence angle on shallow water. Their asymptotic decomposition is exactly as in figure 7 , except that, relative to the moving frame, there is uniform flow behind the impactor with velocity $-U$ and height $1-s_{0}$. We therefore conjecture that the unsteady planing model (17 - 22), with $s(t) \rightarrow s_{0}$ as $t \rightarrow \infty$, tends to the travelling wave solution of [11] sufficiently near to the plate with $d_{0}=\lim _{t \rightarrow \infty} d(t)$. The flow in region III behind the impactor tends to uniform flow with velocity $-U$ (and height $1-s_{0}$ ), so that $\dot{c} \rightarrow 0$ as $t \rightarrow \infty$ by (22). This implies that the flux ejected into the spout tends to zero as $t \rightarrow \infty$ and that our asymptotic model will eventually break down whenever gravity is present.

\subsubsection{Shallow water grazing of a finite flat plate at zero deadrise angle}

In this section we describe case (4) for the special case in which a flat finite plate grazes shallow water with zero deadrise angle and at constant velocity. The normal impact case was analysed by Korobkin [17]: assuming that the free surface separates smoothly from the ends of the plate, he showed that two symmetric spouts are ejected in exactly the same way as the downstream spout in figure 7 . We employ the notation of the case (4) grazing model in $\S 3.1$, so that the plate has equation $f\left(x^{*}\right)=0$ for $\left|x^{*}\right|<1$, constant normal impact velocity $\dot{s}=1$ and constant tangential velocity $U^{*}=U / \epsilon$. For reasons that will become apparent, we anticipate that the flow morphology depends crucially on whether $U$ is greater or less than the critical tangential velocity $U=1 / 2$ as illustrated in figure 8 .

The inviscid squeeze film flow beneath the plate is again governed by (15). Scaling $x^{*}=x / \epsilon$ and $\phi^{*}=\phi / \epsilon^{2}$ as in $\S 3.1$, the trailing $(-)$ and leading $(+)$ end of the plate 

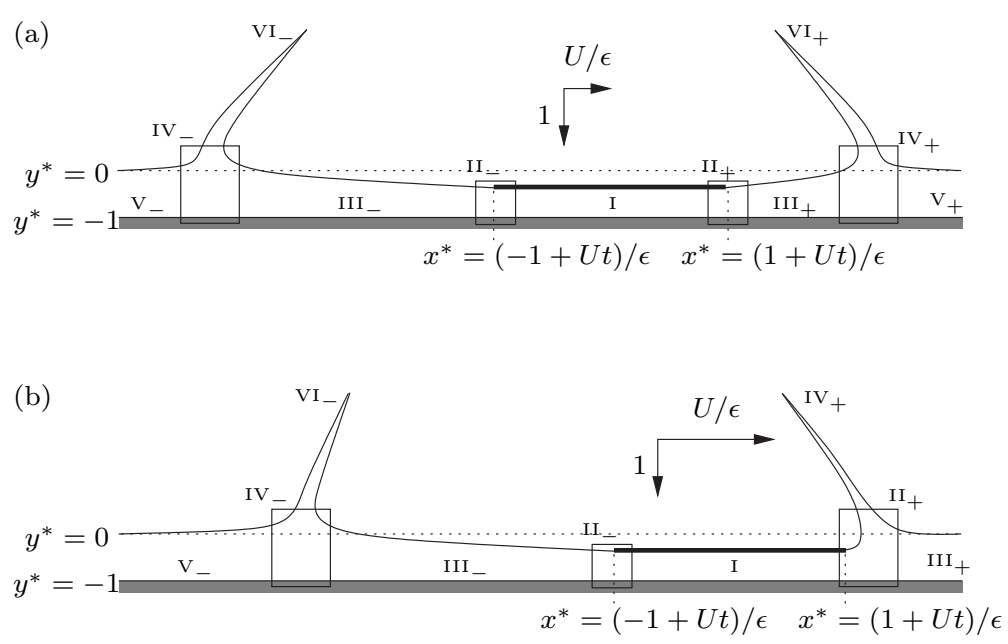

Figure 8: Proposed case (4) flow geometries; (a) detached leading spout $(U<1 / 2)$ and (b) attached leading spout $(U>1 / 2)$.

becomes $\left(x_{ \pm}(t),-t\right)$, where $x_{ \pm}(t)= \pm 1+U t$, and the leading order potential $\phi(x, t)$ is

$$
\phi(x, t)=A+\frac{\left(x-x_{0}\right)^{2}}{2(1-t)},
$$

where $A(t)$ and the location of the relative stagnation point $x=x_{0}(t)$ are to be determined.

At sufficiently small tangential velocities $U$, which we quantify below, fluid is ejected from both ends of the plate and the flow structure is therefore exactly as in the normal impact case as depicted in figure $8(\mathrm{a})$. Continuity of pressure across regions $\mathrm{II}_{ \pm}$in figure 8(a) implies

$$
p=-\phi_{t}-\frac{1}{2} \phi_{x}^{2}=0
$$

at $x=x_{ \pm}$, and therefore

$$
x_{0}=U t^{2}, \dot{A}=\frac{(1-t)\left(x-x_{0}\right) \dot{x}_{0}-\left(x_{-}-x_{0}\right)^{2}}{(1-t)^{2}} .
$$

The fluid is therefore ejected from the edges of the plate with relative velocity

$$
\phi_{x}\left(x_{ \pm}, t\right)-U= \pm \frac{1}{1-t}-U(1-t) .
$$

Hence, there is always outflow at the trailing edge and the trailing regions $\mathrm{II}_{-}-\mathrm{VI}_{-}$in figure 8 are the same as the trailing regions II-VI in figure 7 . However, at the leading edge there is outflow if and only if $t>\max \left(0,1-U^{-1 / 2}\right)$. Since there is initially outflow at the leading edge if and only if $U<1$, one might expect Korobkin's 'detatched spout' decomposition to be valid when $U<1$. However, the jump condition (22) says that a spout root moves with half the velocity of the incoming fluid velocity of the neighbouring shallow water region $\left(\mathrm{III}_{ \pm}\right.$in figure $\left.8(\mathrm{a})\right)$. Furthermore, the initial velocity of the ejected streams is $\phi_{x}( \pm 1,0)= \pm 1$, so the spout root always escapes from the trailing edge, but cannot escape from the leading edge unless $U<1 / 2$. Hence, if $U>1 / 2$, the tangential velocity is sufficiently large that the inviscid squeeze film flow in region I cannot initially 
cause the formation of a detached spout at the leading edge. Korobkin's decomposition then becomes invalid and instead we envisage the 'ploughing' scenario depicted in figure 8(b), in which the spout is fixed at the leading edge in the 'gap-sized' region $\mathrm{II}_{+}$. Assuming exponentially small flow in the region $\mathrm{III}_{+}$ahead of the plate, we do not need to use the exact solution to the resulting leading-order Kelvin-Helmholtz cavity flow in region $\mathrm{II}_{+}$ (due to Green [9]) because the leading-order Bernoulli equation there implies that the pressure matching condition at the leading edge becomes

$$
p+\frac{1}{2} \phi_{x}^{2}=\frac{1}{2} U^{2} \quad \text { at } \quad x=x_{+} .
$$

Moreover, the ejected spout speed is $U$ and its thickness and direction are determined by global conservation of mass and horizontal momentum in region $\mathrm{II}_{+}$. Substituting (23) into (24) at $x=x_{-}$and (26) we find

$$
4(1-t)\left(\dot{x}_{0}-U\right)+(6+2 U(1-t))\left(x_{0}-U t\right)+\left(x_{0}-U t\right)^{2}=2 U(1-t)-1,
$$

with $\dot{A}$ given by (25). Solving numerically for a range of $U$, we find that there is always outflow at the trailing edge. We plot the relative velocity at the leading edge for $U=1 / 4$ and 4 in figures $9(\mathrm{a}, \mathrm{b})$, which are typical for the cases $U<1 / 2$ and $U>1 / 2$. In fact there is outflow at the leading edge for all $t>\max \left(0, t_{1}(U)\right)$ where $t_{1}(U)$ is such that $\phi_{x}\left(x_{+}\left(t_{1}\right), t_{1}\right)=U$ and is plotted in figure $9(\mathrm{c})$. This suggests that the ploughing solution is valid for all $U>0$, so it appears that there are two solutions for $0<U<1 / 2$.

One mechanism for selecting the physically acceptable solution comes from consideration of the pressure. In figures $9(\mathrm{a}, \mathrm{b})$ we also plot the leading-order pressure at the leading edge, $p\left(x_{+}, t\right)$, for $U=1 / 4$ and 4 , which are again typical for the cases $U<1 / 2$ and $U>1 / 2$. In fact, the pressure at the leading edge is equal to $\frac{1}{2}\left(U^{2}-(1-U)^{2}\right)$ at $t=0$, so the ploughing scenario predicts that the flow near the leading edge will cavitate immediately after impact if $U<1 / 2$. Morover, if $U>1 / 2$, the flow does not cavitate immediately, but must do so near the leading edge at the time $t=t_{2}(U)$ at which the pressure vanishes. By $(26), \phi_{x}\left(x_{+}\left(t_{2}\right), t_{2}\right)=2 U$, which we use to plot $t_{2}(U)$ in figure $9(\mathrm{~d})$. Hence, if we demand that the pressure is non-negative everywhere, we immediately rule out the ploughing solution for the case $U<1 / 2$.

The only leading-order inner flow at an end of the plate that maintains a zero pressure matching condition for the lowest-order flow beneath the impactor is uniform flow (as in regions $\mathrm{II}_{ \pm}$in figure $\left.8(\mathrm{a})\right)$. In the case $U>1 / 2$, we therefore envisage a transition to the detached spouting regime (in figure 8(a)) at time $t=t_{2}(U)$, when the pressure at the leading edge vanishes. Since the attached spout $\mathrm{II}_{+}$cannot escape from the leading edge unless $\phi_{x}\left(x_{+}, t\right) \geq 2 U, t=t_{2}(U)$ is also the earliest time at which the transition can occur.

After the proposed change in the flow regime, the $x$-coordinate of the relative stagnation point $x_{0}(t)$ is given by $(22)$. If we demand $x_{0}$ to be continuous across the jump, then so are the lowest order inviscid squeeze film velocity and pressure, although there is a discontinuity in their time derivatives.

\section{Three-dimensional generalizations}

The three-dimensional generalizations of the grazing flows in $\S 2.1$ and $\S 3.1$ are straightforward. Denoting the third space direction by $\eta$ in the model in figure 2, we simply 

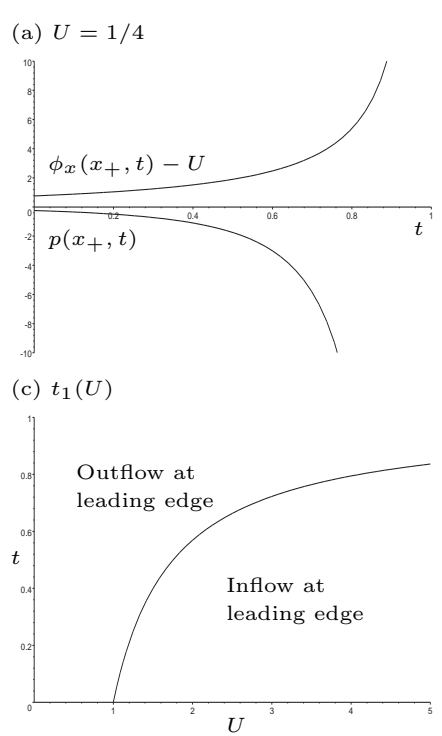
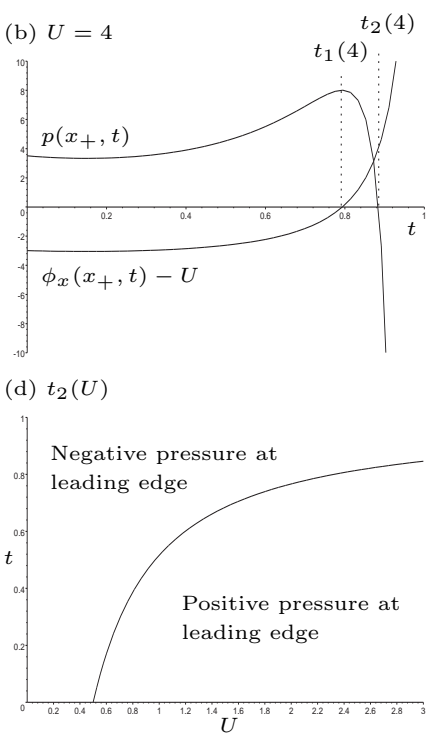

Figure 9: Plots of the relative fluid velocity and pressure at the leading edge for (a) $U=1 / 4$ and (b) $U=4$. Plot of the critical times (c) $t_{1}(U)$ and (d) $t_{2}(U)$.

replace $f(x)$ by $f(x, \eta), h(x, t)$ by $h(x, \eta, t)$ and allow $d_{ \pm}$to depend on $\eta$ and $t$. The local behaviour near the turnover region is as in the two-dimensional case and the Wagner condition is unaltered. Although we cannot use complex variables as in $\S 2.1$, we can still use the variational formulation in figure 3 to make analytic and numerical progress.

Similar remarks apply to the case (2) scenario where $H=\mathrm{O}(1)$ and in the small- $H$ limit we anticipate that (12) will become

$$
\iint_{\mathcal{I}(t)}(s(t)-f(\xi-U t, \eta)) k(\xi, \eta) \mathrm{d} \xi \mathrm{d} \eta=0 \quad \text { for } \quad k(\xi, \eta)=1, \xi, \eta
$$

where the integral is taken over the "inviscid squeeze film" region $\mathcal{I}(t)$ and represents conservation of mass, $\xi$-momentum and $\eta$-momentum, respectively. Moreover, the threedimensional generalization of the case $(4)$ governing equations $(15,16)$ is clearly

$$
F_{t}+\left(F \phi_{x}\right)_{x}+\left(F \phi_{y}\right)_{y}=0
$$

in the leading order squeeze film, with

$$
\phi=0, \phi_{\nu}=2 v_{\nu}\left(1-F^{-1 / 2}\right),
$$

on the boundary $\partial \mathcal{I}(t)$ of $\mathcal{I}(t)$, which is now the "turnover curve"; the base-to-body height is $F(x, \eta, t)=1+f(x-U t, \eta)-s(t)$, the subscript $\nu$ denotes the outward normal derivative on the turnover curve and $v_{\nu}$ its outward normal velocity. The small-time limit of this novel free boundary problem is the generalized Hele-Shaw problem

$$
\begin{gathered}
\nabla^{2} \phi=\dot{s}+U f_{x}(x-U t, \eta) \quad \text { for } \quad(x, \eta) \in \mathcal{I}(t) \\
\phi=0, \phi_{\nu}=v_{\nu}(f(x-U t, \eta)-s(t)) \quad \text { for } \quad(x, \eta) \in \partial \mathcal{I}(t) .
\end{gathered}
$$


Here we simply note that it is straightforward to show that the generalized Richardson moments are conserved:

$$
\left.\iint_{\mathcal{I}(t)}(s(t)-f(x-U t, \eta)) \psi\right)(x+i \eta)^{j} \mathrm{~d} x \mathrm{~d} \eta=0 \quad \text { for } \quad j=0,1, \ldots
$$

which confirms (27).

The three-dimensional generalizations of the planing models in $\S 2.2$ and $\S 3.2$ are even more difficult because (i) the location of the transition points at the intersection of the turnover curve and the trailing edge and (ii) the nontrivial interaction between the fluid 'ejected' from the trailing edge and the fluid behind the planing body must also be determined; see [18, pp 363-370] for a discussion of the steady infinite depth case. However, in the skimming regime of case (4), the flows in the interior region (between the turnover curve and the trailing edge) and in the exterior region (outside the interior region) decouple, as we now describe for the steady case. We introduce the horizontal $\eta^{*}$ coordinate and suppose that the convex planing body has equation $y^{*}=f\left(\epsilon \xi^{*}, \epsilon \eta^{*}\right)-s_{0}$ relative to a frame moving with speed $U$ in the $\xi^{*}$-direction, where $f(0,0)=0$ and the penetration depth $s_{0}=\mathrm{O}(1)$. For small incidence angles $\epsilon \ll 1$, the flow decomposes into the three outer regions of horizontal extent $\mathrm{O}(1 / \epsilon)$ in the starred coordinates shown in figure 10 for a circular skimboard.

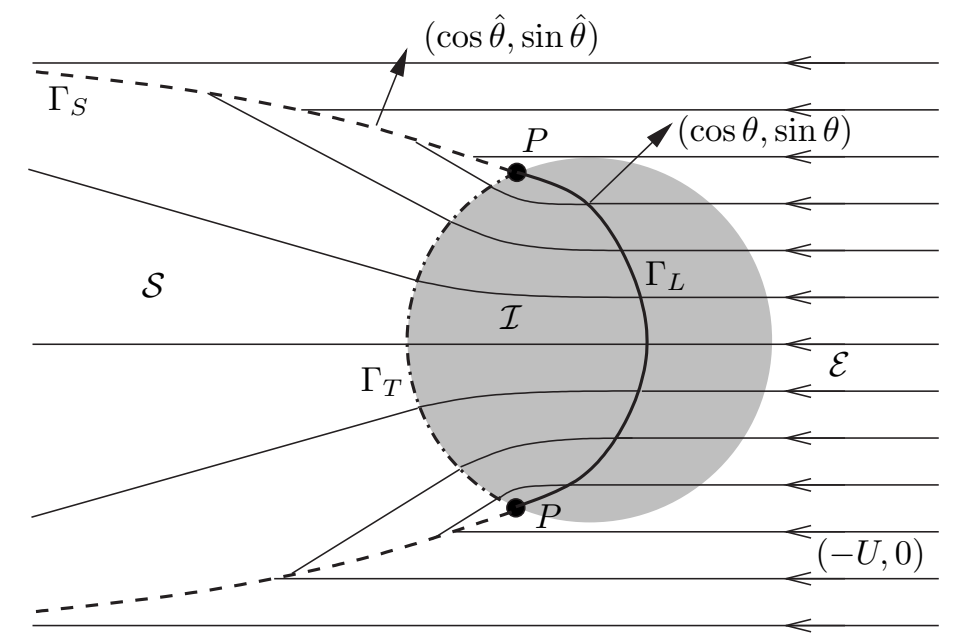

Figure 10: Plan view of the three outer regions for three-dimensional unsteady skimming and a possible streamline configuration for a circular skimboard (shaded region). The interior region $\mathcal{I}$ is under the body between the turnover curve $\Gamma_{L}$ and the trailing edge $\Gamma_{T}$. The transition points labelled $P$ lie at the intersection of $\Gamma_{L}$ and $\Gamma_{T}$. The trailing stream region $\mathcal{S}$ consists of the fluid ejected from the trailing edge $\Gamma_{T}$, which impacts with the uniform flow $(-U, 0)$ in the exterior region $\mathcal{E}$ at the spout root $\Gamma_{S}$. Across $\Gamma_{S}$, the streamlines are discontinuous because all the fluid entering from either side of $\Gamma_{S}$ is lost to the ejected spout.

In the exterior region $\mathcal{E}$, a streamline of the incoming uniform flow $(-U, 0)$ ahead of the skimmer either hits the turnover curve $\Gamma_{L}$, a transition point $\mathrm{P}$, the trailing stream $\mathcal{S}$ at the spout root $\Gamma_{S}$, or possibly nothing. 
In the interior region $\mathcal{I}$, the three-dimensional steady generalization of $(17-19)$ is (scaling $\left(\xi^{*}, \eta^{*}\right)=\epsilon^{-1}(\xi, \eta)$ and $\left.\phi=\epsilon^{-2} \tilde{\phi}\right)$

$$
\begin{gathered}
\nabla \cdot(\tilde{F} \nabla \tilde{\phi})=0 \quad \text { for } \quad(\xi, \eta) \in \mathcal{I}, \\
\tilde{\phi}+U \xi=0, \tilde{\phi}_{\nu}=U \cos \theta\left(1-2 \tilde{F}^{-1 / 2}\right) \text { for }(\xi, \eta) \in \Gamma_{L}, \\
|\nabla \tilde{\phi}|=U \text { for }(\xi, \eta) \in \Gamma_{T},
\end{gathered}
$$

where $\tilde{F}(\xi, \eta)=1+f(\xi, \eta)-s_{0}$ is the base-to-body height, $\tilde{\phi}_{\nu}$ is the outward normal derivative of $\tilde{\phi}$ on the turnover curve $\Gamma_{L}$, which has outward unit normal $(\cos \theta, \sin \theta)$.

In the trailing stream $\mathcal{S}$ ejected from the trailing edge $\Gamma_{T}$, the three-dimensional steady generalization of the zero-gravity shallow water equations (20) for the leadingorder potential $\hat{\phi}(\xi, \eta)$ and free surface $y^{*}=\hat{h}(\xi, \eta)$ are

$$
|\nabla \hat{\phi}|=U, \nabla \cdot(\hat{h} \nabla \hat{\phi})=0 \quad \text { for } \quad(\xi, \eta) \in \mathcal{S},
$$

while the boundary data (21) become

$$
\hat{\phi}=\tilde{\phi}, \hat{h}=\tilde{F} \quad \text { for } \quad(\xi, \eta) \in \Gamma_{T} .
$$

The trailing stream $\mathcal{S}$ impacts with the uniform flow $(-U, 0)$ in the exterior region $\mathcal{E}$ forming a 'spout sheet' that is ejected from the root $\Gamma_{S}$, in which the flow is quasitwo-dimensional in planes perpendicular to $\Gamma_{S}$ and whose cross-sections have the same structure as region IV in figure 7 . The steady generalization of (22) is

$$
\hat{\phi}_{\nu}=U \cos \hat{\theta} \quad \text { for } \quad(\xi, \eta) \in \Gamma_{S},
$$

where $\hat{\phi}_{\nu}$ is the outward normal derivative of $\hat{\phi}$ on the spout root $\Gamma_{S}$, which has outward unit normal $(\cos \hat{\theta}, \sin \hat{\theta})$. Hence, the spout root bisects the angle between the straight incoming streamlines from the trailing stream $\mathcal{S}$ and the exterior region $\mathcal{E}$. Moreover, it is straightforward to show from $(29,30)$ that $|\nabla \tilde{\phi}|$ is continuous at a transition point $P$ if and only if $\cos \theta(P)=0$ or $\tilde{F}(P)=1$. In the former case $P$ lies at an extremity of the body, while in the latter case the base-to-body height at $P$ is equal to the undisturbed water level. We conjecture that the latter is selected because the spout sheet thickness is zero at a transition point if and only if the base-to-body height there is equal to the undisturbed water level. It would be interesting to see if the predicted location of the spout root is similar to those observed in practice as shown in figure 11. Finally, we remark that, in the absence of gravity, the spout centre-surface is a ruled surface determined by the velocity of the fluid particles ejected from the spout root, while its thickness is determined by the usual conservation of mass argument for a thin sheet.

\section{Conclusions}

We have presented a framework within which to link the normal-impact theories of Wagner and Korobkin to the theories of planing and skimming. We have shown that, even in two dimensions, there are open questions concerning "downstream" separation in cases where the angle of impact is of the order of the deadrise angle; for a symmetric wedge impact, an instability arises when the impact angle is about one-half the deadrise angle. We have also shown that three-dimensional flows present many interesting challenges, especially for the modelling of the spray sheets that are found in shallow-water skimming. 


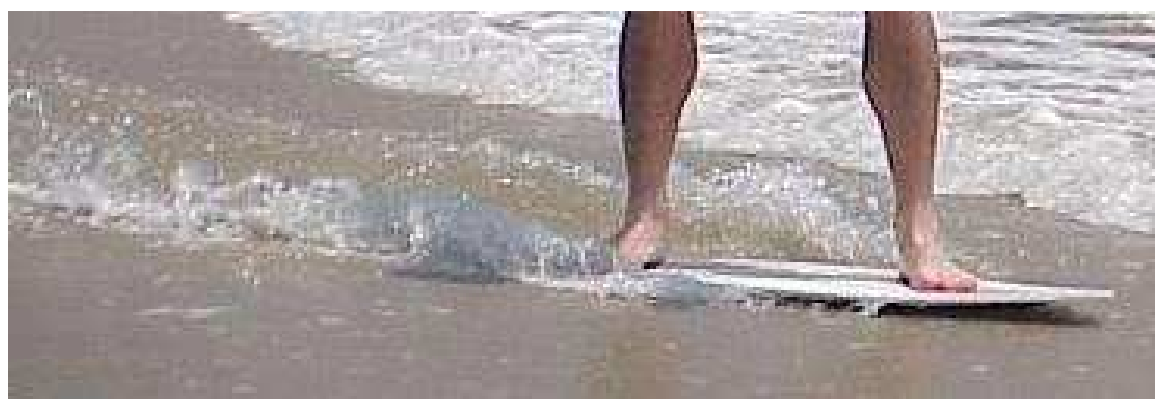

Figure 11: Surf-skimming.

\section{Acknowledgements}

We are grateful for helpful comments from A.A. Korobkin and E.O. Tuck. J.M.O. wishes to thank the EPSRC for financial support via a studentship.

\section{References}

[1] S.D. Howison, J.D. Morgan, and J.R. Ockendon. Codimension-two free boundary problems. SIAM Review, 39(2):221-253, 1997.

[2] H. Wagner. Über stoß- und gleitvorgänge an der oberfläche von flüssigkeiten (Phenomena associated with impacts and sliding on liquid surfaces). Zeitschrift für Angewandte Mathematik und Mechanik, 12:193-215, 1932.

[3] J.M. Oliver. Water-entry and related problems, 164 pp. PhD thesis, Oxford, 2002.

[4] S.D. Howison, J.R. Ockendon, and J.M. Oliver. Deep- and shallow-water slamming at small and zero deadrise angles. J. Engg. Math, 42:373-388, 2002.

[5] A.A. Korobkin. Impact of two bodies one of which is covered by a thin layer of liquid. J. Fluid Mech., 300:43-58, 1995.

[6] L.M. Trefethen and R.L. Panton. Some unanswered questions in fluid mechanics. Appl. Mech. Rev., 43(8):153-170, 1990.

[7] A.A. Korobkin. Formulation of penetration problem as a variational inequality. Din. Sploshnoi Sredy, 58:73-79, 1982.

[8] S.D. Howison, J.R. Ockendon, and S.K. Wilson. Incompressible water-entry problems at small deadrise angle. J. Fluid Mech., 222:215-230, 1991.

[9] A.E. Green. The gliding of a plate on a stream of finite depth. Proc. Cam. Phil. Soc., 31:589-603, 1935.

[10] S. Ulstein and O.M. Faltinsen. Two-dimensional unsteady planing. J. Ship Res., 40(3):200-210, 1996. 
[11] E.O. Tuck and A. Dixon. Surf-skimmer planing hydrodynamics. J. Fluid Mech., 205:581-592, 1989.

[12] A.A. Korobkin. Inclined entry of a blunt profile into an ideal fluid. Izv. Akad. Nauk SSSR, Mekh. Zhidk. Gaza, pages 145-150, 1988.

[13] A.B. Tayler. Singularities at flow separation points. Quart. Journ. Mech. and Appl. Math., XXVI(2):153-171, 1972.

[14] G.M. Fridman. Matched asymptotics for two-dimensional planing hydrofoils with spoilers. J. Fluid Mech., 358(259-281), 1998.

[15] L. Ting and J.B. Keller. Planing of a flat plate at high Froude number. Phys. Fluids, 17:1080-1086, 1974.

[16] Y.T. Wu. A singular perturbation theory for nonlinear free surface flow problems. Intl. Shipbuilding Proc., 14:88-97, 1967.

[17] A.A. Korobkin. Shallow-water impact problems. J. Eng. Math., 35:233-250, 1999.

[18] E.O. Tuck. Numerical Solution of Integral Equations, xiii +417 pp, chapter 7, "Planing Surfaces", pages 363-372. Plenum Press, New York, 1990. 